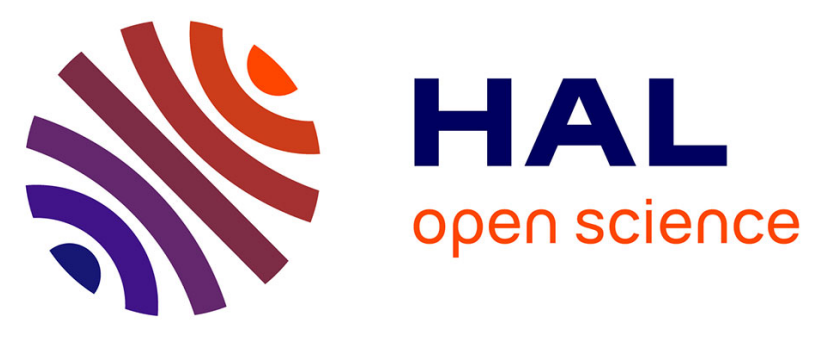

\title{
Native Mass Spectrometry, Ion Mobility, and Collision-Induced Unfolding for Conformational Characterization of IgG4 Monoclonal Antibodies
}

Oscar Hernandez-Alba, Elsa Wagner-Rousset, Alain Beck, Sarah Cianférani

\section{- To cite this version:}

Oscar Hernandez-Alba, Elsa Wagner-Rousset, Alain Beck, Sarah Cianférani. Native Mass Spectrometry, Ion Mobility, and Collision-Induced Unfolding for Conformational Characterization of IgG4 Monoclonal Antibodies. Analytical Chemistry, 2018, 90 (15), pp.8865-8872. 10.1021/acs.analchem.8b00912 . hal-02327964

\section{HAL Id: hal-02327964 \\ https://hal.science/hal-02327964}

Submitted on 8 Oct 2020

HAL is a multi-disciplinary open access archive for the deposit and dissemination of scientific research documents, whether they are published or not. The documents may come from teaching and research institutions in France or abroad, or from public or private research centers.
L'archive ouverte pluridisciplinaire HAL, est destinée au dépôt et à la diffusion de documents scientifiques de niveau recherche, publiés ou non, émanant des établissements d'enseignement et de recherche français ou étrangers, des laboratoires publics ou privés. 


\title{
Native Mass Spectrometry, lon Mobility and Collision Induced Un- folding for Conformational Characterisation of $\operatorname{lgG}_{4}$ Monoclonal An- tibodies
}

\author{
Oscar Hernandez-Alba ${ }^{1}$, Elsa Wagner-Rousset ${ }^{2}$, Alain Beck², Sarah Cianférani ${ }^{*}$ \\ 1 Laboratoire de Spectrométrie de Masse BioOrganique, Université de Strasbourg, CNRS, IPHC UMR 7178, 67000 \\ Strasbourg, France \\ 2 IRPF - Centre d'Immunologie Pierre-Fabre (CIPF), 7416o Saint-Julien-en-Genevois, France
}

\begin{abstract}
Although the majority of FDA and EMA approved therapeutic monoclonal antibodies (mAbs) are IgGr, the number of IgG4-based formats reaching the market is increasing. IgG 4 differ from other $\mathrm{mAb}$ isotypes by its specificity to form half mAbs that recombine into bispecific (bsAbs) molecules, through a process termed fab-arm exchange (FAE). We report here the complementarity of native mass spectrometry (MS), ion mobility (IM) and collision induced unfolding (CIU) experiments for the structural characterization of member IgG4 subfamily (wild-type (wt), hinge-stabilized (hs, S228P mutation) and the resulting bsAb IgG4s)). Native MS allows confirming/invalidating the occurrence of FAE as a function of these different types of IgG4. While IM-MS was unable to distinguish iso-cross-section IgG4 species, CIU experiments provide unique specific structural signatures of each individual $\mathrm{IgG}_{4}$ based on their specific unfolding pathways. Common CIU features of $\mathrm{IgG}_{4}$ formats include the observation of three conformational states and two transitions. In addition, CIU experiments S228P mutation stabilized gas phase conformations of hsIgG4, in agreement with increased stability related to more rigid hinge regions. CIU patterns also appear to be more informative than IM-MS for bsAb structural characterization, unfolding signature of the bsAb being intermediate to the ones of the former parent wtIgG4s, highlighting that bsAb CIU profiles keep the memory of their origins. Altogether, our results demonstrate that CIU patterns can serve as mAb specific structural signatures and are mature to be included in MS-based analytical workflows for conformational/structural characterization of $\mathrm{mAb}$ formats in early development phases and for multiple attribute monitoring.
\end{abstract}

\section{Introduction}

Monoclonal antibody-based therapeutics (mAbs) are the most promising drug class for clinical treatment of cancers, autoimmune diseases, cardiovascular disorders, ophthalmic diseases or asthma ${ }^{1-3}$. More than 70 mAbs and related products have been approved by the Food and Drug Administration (FDA) and by the European Medicine Agency (EMA) ${ }^{4}$, with more than 500 currently in clinical development ${ }^{1,5}$. Since the mid-1990s, different strategies have been developed in order to produce more efficient and specific antibody-based therapeutics, giving rise to a plethora of mAbs and related products, such as antibody drug conjugates $(\mathrm{ADCs})^{6}$, Fab fragments, radioimmunoconjugates ${ }^{7}$, and bispecific antibodies (bsAb) . $^{8}$

Most currently approved mAbs are selected from three human IgG isotypes (1, 2 or 4$)$, which are defined by different heavy-chain amino acid sequences ${ }^{9}$, the total number of disulfide bridges ( 16 for IgG1 and IgG4 and 18 for $\mathrm{IgG2}$ ) and disulfide bond connectivities between heavy chains in the flexible hinge region (two for IgGr and $\mathrm{IgG}_{4}$, four for IgG2). Currently, the majority of mAbs and ADCs approved by the FDA and the EMA are based on the IgG1 isotype (chimeric, humanized or human) ${ }^{10}$, while $\operatorname{IgG} 3$ is not used in the form of therapeutics owing to a significantly faster clearance rate (up to three times faster). IgG1 is considered to be easier to develop than IgG2 (more complex hinge region) ${ }^{11}$ or wt-IgG4. Human IgGs of different isotypes also differ in their ability to support secondary immune functions: $\operatorname{IgG} 1$ can usually support antibody-dependent cellular cytotoxicity (ADCC) and complement-dependent cytotoxicity (CDC), whereas IgG2 and $\mathrm{IgG}_{4}$ are typically inefficient or limited in their effector functions ${ }^{12}$. Human $\mathrm{IgG}_{4}$ may sometimes support antibody-dependent cellular phagocytosis (ADCP) ${ }^{13}$. The number of $\operatorname{IgG}_{4}$-based therapeutics reaching the market and in clinical trials is increasing progressively ${ }^{14}$, serving as the basis for a variety of $\mathrm{mAb}$ formats including $A D C \mathrm{~s}^{10}$, naked mAbs, PEGylated Fabs ${ }^{15}$, Fc-fusion peptides ${ }^{16}$ and $\operatorname{bsAbs}^{17}$ (see Supporting Information Table Si). The interest in $\mathrm{IgG}_{4} \mathrm{mAbs}$ in the biopharmaceutical field stems from its ability to form half mAbs (one heavy and one light chain; $75 \mathrm{kDa})^{18}$ and bsAbs in vitro and in vivo (through a process termed Fab-arm exchange (FAE) when not stabilized by a serine-to-proline mutation in the hinge region ${ }^{19}$. BsAbs are thus able to bind to two different types of antigens ${ }^{20}$. Natalizumab, indicated for multiple sclerosis, and more 
recently reslizumab, intended for severe asthma, are examples of so-called wild-type (wt) therapeutic $\operatorname{IgG} 4 \mathrm{~s}$. This particular characteristic of $\mathrm{IgG}_{4} \mathrm{mAbs}$ can be explained by different determinants in the primary structure compared to other IgGs; namely, the flexibility associated with the $\mathrm{IgG}_{4}$ hinge region (shorter than $\mathrm{IgG1}$ ), the relatively labile disulfide bonds between the two heavy chains in the hinge region, and the non-covalent interactions between the two $\mathrm{CH}_{3}$ domains of the two heavy chains facilitate the H-L pair exchange between two IgG4S (Figure 1) ${ }^{19}$. As pharmacokinetics and pharmacodynamics of wt-IgG4 mAbs can vary as a function of FAE and lead to undesired clinical effects, biopharmaceutical companies have made remarkable progress in developing more stabilized $\mathrm{IgG}_{4}$ formats, by introducing S228P point mutation in the core-hinge sequence of $\mathrm{IgG}_{4} \mathrm{mAbs}$ that avoids FAE ${ }^{21}$. Currently, most of the biopharmaceutical companies are using such hinge stabilized $\mathrm{IgG}_{4}$ versions in order to abrogate FAE and address regulatory authority safety concerns ${ }^{22}$. Pembrolizumab $^{23}$ and nivolumab ${ }^{24}$ are two examples of such hinge-stabilized engineered $\mathrm{IgG}_{4}$ formats that have been approved for cancer treatment ${ }^{25}$.

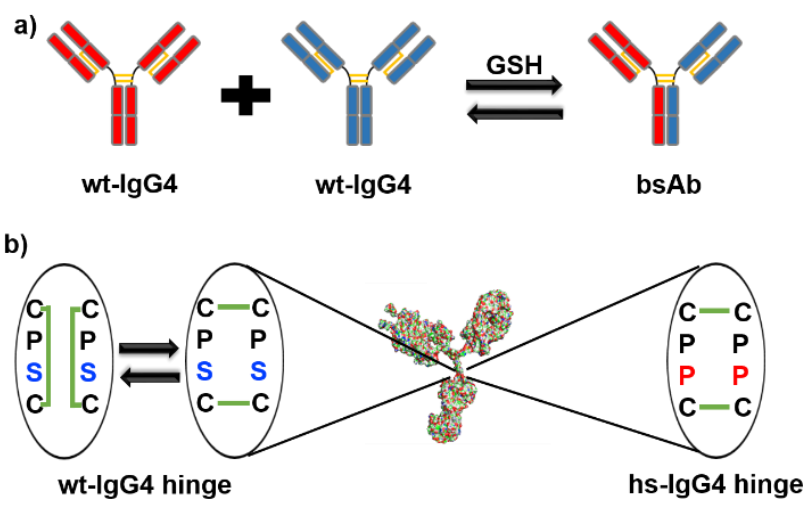

Figure 1. a) Schematic representation of FAE reaction between two wt-IgG4s. b) Difference between the corehinge region of wt-IgG4 (left) and hs-IgG4 mAbs (right).

Using native mass spectrometry, which has been demonstrated to be mature enough to be integrated into routine analytical workflows for $\mathrm{mAb}$ homogeneity assessment and the critical quality attribute determination of $\mathrm{mAbs}^{26-29}$, we aimed to promote the use of native ion mobility mass spectrometry (IM-MS) along with gas-phase collision-induced unfolding (CIU) for their structural characterization. CIU approaches have been reported to circumvent poor IM separation of co-migrating species that exhibit very close structures ${ }^{30-31}$. In particular, Tian et al. ${ }^{22}$ demonstrated that CIU experiments allow for distinguishing the four different $\mathrm{mAb} \operatorname{IgG}$ isotypes, showing that IM migration times and the unfolding transitions strongly depend on the number of disulfide bond patterns within the isotype structures of the mAbs.

In the present work, we focus on the combination of advanced native MS and IM-MS approaches for the structural characterization of different therapeutic $\mathrm{IgG}_{4}$ formats; namely, two wt-IgG4s (natalizumab and reslizumab), two hinge stabilized (hs) hs-IgG4s (pembrolizumab and nivolumab) and the bsAb formed after FAE between wtIgG4s. Native MS allows for confirming/infirming the occurrence of FAE and subsequent bsAb formation as a function of the $\mathrm{IgG}_{4}$ format (wt versus hs). The global characterization of wt-IgG4 and hs-IgG4s and ${ }^{{ }^{T}{ }^{2}} \mathrm{CCS}_{\mathrm{N}_{2}}$ measurements were performed by IM-MS, along with experiments involving CIU, as the specific signature of individual therapeutic protein, revealing that hs-IgG4 constructs are less prone to unfolding than wt-IgG4, in agreement with increased gas-phase stability related to hinge region rigidity. The CIU signature of FAE-produced bsAb also clearly demonstrate that its gas-phase stability is intermediate between parent $I_{g} G_{4}$ s. Altogether, our results demonstrate that CIU experiments are unique fingerprints of biologics.

\section{Materials and methods}

FAE reaction natalizumab/reslizumab bsAb formation.

Different mixtures of two IgG 4 mAbs were used to form bsAb. Either two wt-IgG4 mAbs (natalizumab and reslizumab), or one wt-IgG4 (natalizumab) and one hs$\mathrm{IgG}_{4}$ (nivolumab), or two hs-IgG4s (pembrolizumab and nivolumab) were used to produce bsAb through FAE reaction. Endogenous half-molecule swapping conditions were mimicked by using glutathione (GSH, Sigma) as a reducing agent. Initially, parental $\mathrm{mAbs}$ were diluted in $200 \mu \mathrm{l}$ of $1 \times$ PBS solution, leading to a final concentration of $1 \mathrm{mg} / \mathrm{ml}$ for each individual antibody. The three different equimolar mixtures of parental $\mathrm{mAbs}$ were subsequently incubated for $24 \mathrm{~h}$ in the presence of $0.5 \mathrm{mM}$ of GSH.

\section{Native MS experiments}

Natalizumab (Tysabri, Biogen), reslizumab (Cinqaero, Teva), nivolumab (Opdivo, BMS) and pembrolizumab (Keytruda, MSD) were obtained from their respective manufacturers. Primary sequences of wt- and hs-IgG4s along with expected masses are provided as Supplementary Information Figure $\mathrm{S}$. Each individual $\mathrm{mAb}$ and all the equimolar mixtures of two $\mathrm{IgG}_{45}$ were $\mathrm{N}$-deglycosylated for $30 \mathrm{~min}$ at $37^{\circ} \mathrm{C}$ with IgGZERO (Genovis) and subsequently desalted against $100 \mathrm{mM}$ of ammonium acetate at $\mathrm{pH} 7$ prior to native MS analysis, using eight cycles of a centrifugal concentrator (Vivaspin, $30 \mathrm{kDa}$ cutoff, Sartorious, Göttingen, Germany).

The concentration of each individual solution after the desalting process was assessed by UV absorbance using a nanodrop spectrophotometer (Thermo Fisher Scientific, France). Prior to native MS analysis, all the samples were diluted in $100 \mathrm{mM}$ of ammonium acetate at $\mathrm{pH} 7$ to a final concentration of $5 \mu \mathrm{M}$. For IdeS (immunoglobulindegrading enzyme of Streptococcus pyogenes) enzymatic treatment, one hundred units of IdeS enzyme (FabRICATOR, Genovis) were added to $100 \mu \mathrm{g}$ of mAbs in $50 \mathrm{mM} \mathrm{Na} 2 \mathrm{HPO}_{4}$ and $150 \mathrm{mM} \mathrm{NaCl}$ at $\mathrm{pH} 6.6$, and the mixture was incubated at $37{ }^{\circ} \mathrm{C}$ for 60 minutes. The Idesdigested $\mathrm{IgG}_{4}$ formats were also desalted against $100 \mathrm{mM}$ of ammonium acetate and subsequently diluted to reach a final concentration of $5 \mu \mathrm{M}$. 
Native mass spectra were recorded either on an Orbitrap Exactive Plus EMR (Thermo Fisher Scientific, Bremen, Germany) or a QTOF (Synapt G2 HDMS, Waters, Manchester, U.K.) mass spectrometer, both coupled to an automated chip-based nanoelectrospray device (Triversa Nanomate, Advion, Ithaca, USA) operating in the positive ion mode. The capillary voltage and the pressure of the nebulizer gas on the Orbitrap Exactive Plus EMR were set at $1.8-1.9 \mathrm{kV}$ and $0.15 \mathrm{psi}$, respectively. Ions were driven through the mass spectrometer with an in-source voltage of $150 \mathrm{eV}$ and then thermalized in the HCD cell at $50 \mathrm{eV}$. The constant $\mathrm{N}_{2}$ pressure in the backing source region, the HCD cell and the orbitrap analyzer were set to $2,10^{-5}$ and $10^{-9}$ mbar, respectively. Native MS data treatment was performed with the BioPharmaFinder 3.o (Thermo Fisher Scientific, Bremen, Germany). Q-TOF parameters were optimized as previously described in Debaene et al 33 . Briefly, the cone voltage of the source interface was set to $120 \mathrm{~V}$ and the backing pressure was increased to 6 mbar to optimize the transmission of the molecular ions. Mass spectra recorded with Synapt G2 platform were analyzed with MassLynx 4.1 (Waters, Manchester, U.K.).

\section{Native IM-MS and CIU experiments}

Ion mobility experiments were performed on a hybrid QTOF mass spectrometer (Synapt G2, Waters, Manchester, UK). The mass spectrometer was carefully parametrized in order to avoid ion activation. The backing pressure was set to 6 mbar and the cone voltage to $80 \mathrm{~V}$ in order to improve the transmission of "native-like" ions. The travelling wave-based ion trap was filled with a continuous Ar flow of $120 \mathrm{~mL} / \mathrm{min}$ and the trap collision energy was set to $4 \mathrm{~V}$. Ions were separated in the TWIMS cell using a constant $\mathrm{N}_{2}$ pressure of $2.4 \mathrm{mbar}$. The IM wave velocity and height were set to $800 \mathrm{~m} / \mathrm{s}$ and $40 \mathrm{~V}$, respectively. IM data were calibrated as described elsewhere ${ }^{34}$. Briefly, three charge state of three external calibrants (concanavalineA, alcohol dehydrogenase and pyruvate kinase) were used to determine the collision cross section of the ion of interest. MassLynx 4.1 was used to perform IM data interpretation. Reported ${ }^{\mathrm{TW}} \mathrm{CCS}_{\mathrm{N}_{2}}$ values correspond to the average ${ }^{\mathrm{TW}} \mathrm{CCS}_{\mathrm{N}_{2}}$ measurement performed in triplicate under the same experimental conditions.

For the CIU experiments, ions were progressively activated in the trap cell by increasing the trap collision voltage in 5 -V steps from o to $200 \mathrm{~V}$ prior to IM separation during 1-minute run. Ion mobility data, specific to each individual charge state, were compiled to give rise to the CIU fingerprints. CIU data, were analyzed with the opensource CIU_Suite software35, allowing the arrival time distribution (ATD) extraction of specific ions at each trap collision voltage. The ATDs were smoothed using a Savitzky-Golay algorithm with a window length of 3 and a polynomial order of 2 . CIU experiments have been performed in triplicate for each individual mAbs leading to a standard deviation lower than $4 \%$, calculated from the CIUSuite_Stat module of CIU_Suite open-source software.

\section{Results and discussion}

Native mass spectrometry of wild-type and hingemodified $\mathrm{IgG}_{4}$ formats

We first used native MS to check the FAE capabilities of our therapeutic mAbs. Figure 2 presents the FAE experiments involving different combinations of wt- and hs-IgG4 mAbs after 24 hours of incubation in the presence of GSH (Materials and methods). As expected, the hybrid bsAb was only formed when two wt-IgG4 mAbs were combined. Three species including the former $\mathrm{mAbs}$ (natalizumab and reslizumab, $146621 \pm 1$ Da and $144260 \pm 5$ Da, respectively) and the additional formation of the bsAb (145441 Da \pm 4 Da) were detected (Figure 2a). At lower m/z, small amounts of natalizumab and reslizumab half mAbs were also observed $(<5 \%)$, pinpointing that our experimental conditions were carefully adapted to minimize wt-IgG4 dissociation (see Supporting Information Figure S2). The relative intensity of natalizumab, reslizumab and the newly formed bsAb revealed that FAE reaction reached equilibrium after a 24$h$ reaction, which is in good agreement with previous results obtained by Debaene et al33. Conversely, only two species corresponding to the parent mAbs were detected in the case of equimolar mixtures of either one wt- and one hs-IgG4 (Figure 2b) or two hs-IgG4s (Figure 2c), as previously reported ${ }^{22,36-37 .}$

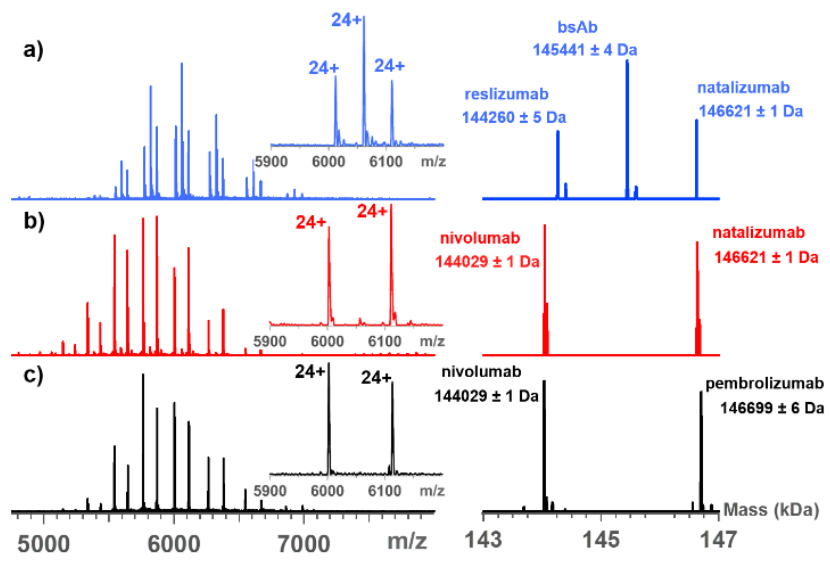

Figure 2. Native mass spectrometry to monitor FAE. Native mass spectra of (a) natalizumab/reslizumab wt-/wtIgG4s, (b) natalizumab/nivolumab wt-/hs-IgG4s and (c) pembrolizumab/nivolumab hs-/hs-IgG4s mixtures obtained after $24^{-h}$ incubation in the presence of GSH. Left panels represent the full-scan native mass spectra and a zoom on the $24+$ charge state as inset; the right panel presents the corresponding deconvoluted mass spectra.

\section{Native IM-MS for the global characterization of $\mathrm{IgG}_{\mathbf{4}}$ formats}

The global conformation of each individual $\mathrm{IgG}_{4}$ was next characterized using native IM-MS. Despite finetuning of IM-MS parameters (see Materials and methods) to provide the best separation of all the species without ion activation, all $\operatorname{IgG}_{4}$ s formats (wt and hs) show very similar 
IM drift times and subsequent ${ }^{{ }^{T W}} \mathrm{CCS}_{\mathrm{N}_{2}}$ (Figure 3). Very similar ATDs can be observed between wt-IgG4s (natalizumab, reslizumab) and hs-IgG4s (pembrolizumab, nivolumab), as depicted on the $22+$ charge state in Figure 3b. For wt-IgG4s, slight ${ }^{{ }^{T W}}{ } C_{4} S_{\mathrm{N}_{2}}$ differences $\left(\sim 0.5 \mathrm{~nm}^{2}\right)$ are observed between natalizumab and reslizumab, which could be attributed to a mass effect, with natalizumab presenting a mass increase of $2 \mathrm{kDa}$ compared to reslizumab (expected CCS increase for $2 \mathrm{kDa}$ is $\left.0.7 \mathrm{~nm}^{2}\right)^{38}$. The same reasons account for the ${ }^{{ }^{T w}} \mathrm{CCS}_{\mathrm{N}_{2}}$ differences observed between pembrolizumab and nivolumab hsIgG4s. The inability of IM-MS to distinguish very close conformations was even more pronounced for higher charge state (Figure 3a). This effect is more likely due to the columbic repulsion as a consequence of the number of protons harbored within the structure of the $\mathrm{IgG}_{4} \mathrm{mAbs}^{39}$. As the studied $\operatorname{IgG}_{4} \mathrm{mAbs}$ share a sequence identity of more than 90\% (Supporting Information table $\mathrm{S}_{2}$ ), possess similar domain structures, all have masses of $\sim 145 \mathrm{kDa}$ and display the same number of disulfide bridges in the hinge region (Figure 1), it was expected to be challenging to distinguish these $\mathrm{IgG}_{4}$ formats based on the sole use of IMMS data due to the current inadaptability between too low resolutive ion mobility cells and resolving power that would be necessary to detect such subtle conformational changes (theoretical IM resolving powers $\Omega / \Delta \Omega>700$ at FWHM would be required to distinguish ${ }^{{ }^{T W}} \mathrm{CCS}_{\mathrm{N}_{2}}$ differences of $0.1 \mathrm{~nm}^{2}$ on intact mAbs). Altogether, these results highlight the difficulty in detecting the subtle conformational variations at the intact $\mathrm{mAb}$ level only by IM-MS on $\mathrm{IgG}_{4}$ formats that have different hinge amino acid sequences with strong impact on biological activities (occurrence of FAE or not for wt-IgG4 and hs$\mathrm{IgG}_{4 \mathrm{~s}}$, respectively).

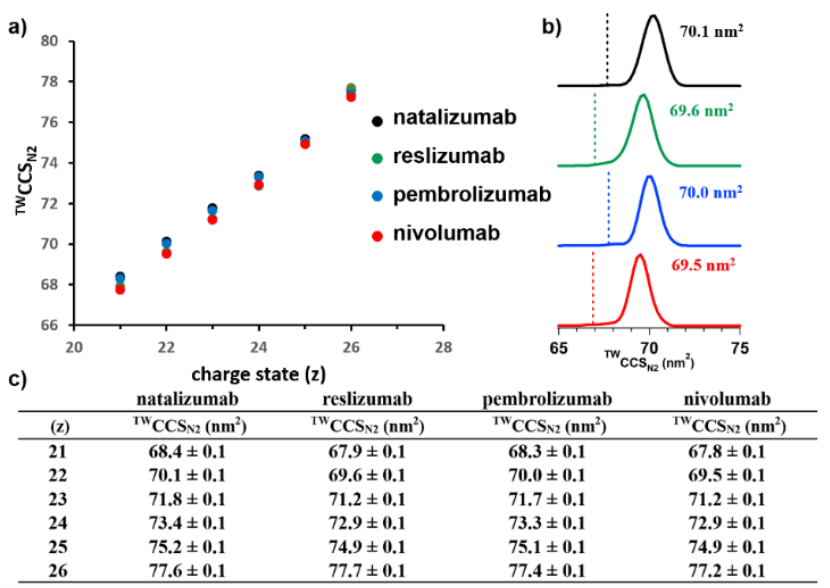

Figure 3. Native IM-MS for conformational characterization of $\mathrm{IgG}_{4}$ formats. (a) ${ }^{\mathrm{Tw}} \mathrm{CCS}_{\mathrm{N}_{2}}$ measurements as a function of the charge state. (b) ATDs of the $22+$ charge state of the four $\mathrm{IgG}_{4}$ formats and comparison with the theoretical ${ }^{\mathrm{TW}} \mathrm{CCS}_{\mathrm{N}_{2}}$ values calculated from $\mathrm{CCS}=2.435^{*}(\mathrm{M})^{(2 / 3)} \quad$ (dashed lines) ${ }^{38}$. (c) Table summarizing all the ${ }^{\mathrm{TW}} \mathrm{CCS}_{\mathrm{N}_{2}}$ measurements with the standard deviation for each individual charge state.
Collision-induced unfolding to monitor $\mathrm{IgG}_{4}$ hinge stabilization

We next performed CIU experiments, a methodology that has been reported to be able to detect subtle conformational changes $3^{3,} 4^{\circ}$, on therapeutic wt(natalizumab and reslizumab) and hs- (pembrolizumab and nivolumab) IgG4. All four mAbs were subjected to gasphase ion activation by increasing the trapping voltage progressively (see Materials and methods). Figure 4 depicts the CIU fingerprints of the $22+$ charge state of the different IgG4s. This charge state was chosen to record the CIU fingerprint taking into account that high charge state can lead to numerous CIU transitions. Three main CIU features are observed for wt-IgG4 (Figure $4 \mathrm{a}$ and $\mathrm{b}$ ): the initial compact state (state o) and two additional unfolded states (state 1 and 2, respectively), in agreement with results reported by Ruotolo's groups on a commercial $\mathrm{IgG}_{4}{ }^{32}$. For both natalizumab and reslizumab, initial compact states have similar lengths and very close centroid IM drift times (16.1 and $15.6 \mathrm{~ms}$, respectively). The first transition from the initial compact state (state o) to the first intermediate state (state 1) occurs between 35 and $40 \mathrm{~V}$. However, clear CIU differences are observed between both wt-IgG4s at higher collision voltages (Figure 4c). The intermediate unfolding state (state 1 ) of natalizumab is observed over a wide range of collision voltages $\left(45^{-200} \mathrm{~V}\right)$, being the most intense conformer from 50 to $140 \mathrm{~V}$. However, state 1 of reslizumab is only the most intense conformer between 45 and $120 \mathrm{~V}$, pinpointing that the latter's intermediate unfolding state 1 undergoes isomerization faster than state 1 of natalizumab. Furthermore, state 2 of natalizumab becomes the most intense structure at $140 \mathrm{~V}$, whereas state 2 of reslizumab can be observed as the most important isomer when the acceleration voltage is set to $125 \mathrm{~V}$. According to our results, the $22+$ charge state of reslizumab is more prone to unfolding due to the fact that the two observed transitions occur at lower accelerating voltages. This behavior is also observed in the CIU fingerprints corresponding to higher charge state (see Supporting Information Figure S3). The reason for differences in reslizumab and natalizumab gasphase sensitivity (both $\operatorname{IgG}_{4}$ s bearing the same number and network of disulfide bonds in the hinge region), may be explained by differences in primary sequences of $m A b s$. To get more details about the mechanism of gas-phase unfolding, we next performed CIU experiments on individual $\mathrm{F}\left(\mathrm{ab}^{\prime}\right)_{2}$ and $\mathrm{Fc}$ fragments (see Supporting Information Figure $\mathrm{S}_{4}$ and $\mathrm{S}_{5}$ ) of both wt-IgG4s generated after IdeS enzymatic treatment (see Materials and methods). IdeS cleaves IgGs between the Fc and F(ab') 2 at a single site at the lower hinge region leaving both fragments intact ${ }^{41}$, generating two domains named $\mathrm{F}\left(\mathrm{ab}^{\prime}\right)_{2}$ and Fc. Almost no differences in CIU patterns could be observed for Fc fragments (see Supporting Information Figure $\mathrm{S}_{5}$ ), in agreement with the high sequence homology (see Supporting Information Table S2) and conserved Fc domain structure for both wt-IgG4s. Conversely, CIU fingerprints originating from $\mathrm{F}\left(\mathrm{ab}^{\prime}\right)_{2}$ domains of reslizumab and natalizumab were significantly different. The same trend is observed for $\mathrm{F}\left(\mathrm{ab}^{\prime}\right)_{2}$ fragments than for 
intact wt-IgG4s, namely that the lightest $\mathrm{F}\left(\mathrm{ab}^{\prime}\right)_{2}$ (96071 Da) of reslizumab is more prone to gas phase unfolding than natalizumab's $\mathrm{F}(\mathrm{ab})^{\prime} \mathbf{2}_{2}$ (98468 Da). As disulfide bridging is a critical quality attribute checked for batch release, we can assume that both mAbs have the same number of disulfide bridges but also the same connectivity in the hinge region. Therefore, these additional CIU experiments allow concluding that $\left.\mathrm{F}(\mathrm{ab})^{\prime}\right)_{2}$ fragments are responsible of the unfolding process differences observed on intact wt-IgG4s.

Altogether, our results clearly demonstrate that the unfolding mechanism of wt-IgG4 $\mathrm{mAbs}$ is driven by $\mathrm{F}\left(\mathrm{ab}^{\prime}\right)_{2}$ domains. As wt-IgG4s share the same number of disulfide bridges in the hinge region and also have the same inter heavy-chain/light chain, differences in primary sequences of $\mathrm{F}\left(\mathrm{ab}^{\prime}\right)_{2}$ domains (including variable domains) might account for the specific gas phase unfolding of each wtIgG4. In addition, we highlight that CIU patterns can act as specific fingerprints/signatures of each individual biotherapeutic.

CIU fingerprints of hs-IgG4 pembrolizumab and nivolumab were very similar to those of wt-IgG4s (Figure 4). Three main CIU features were observed: the initial compact state $o$ and two unfolding states (state 1 and state 2). The centroid IM drift times of both pembrolizumab and nivolumab state o were 16.0 and $15.5 \mathrm{~ms}$, respectively. The collision energy characteristic of two unfolding transitions and the IM drift times are rather similar for both hs-IgG4 formats. Thus, the first transition can be observed at $45 \mathrm{~V}$ (state o to state 1 ), with both pembrolizumab and nivolumab undergoing complete unfolding into state 2 between 75 and $200 \mathrm{~V}$. Conversely to wt-IgG4s, a detailed comparison of both CIU fingerprints does not reveal any significant conformational difference (Figure $4 \mathrm{f}$ ). Similarly to wt-IgG4s, Fc fragments of hs-IgG4s obtained after IdeS digestion were not significantly different while $F(a b ')_{2}$ fragments allow distinguishing the two hs-IgG4s, highlighting again that $\mathrm{CIU}$ pattern of $\mathrm{F}\left(\mathrm{ab}^{\prime}\right)_{2}$ fragment act as signature of each $\mathrm{mAb}$ (see Supporting Information Figure $\left.\mathrm{S}_{5}\right)$.

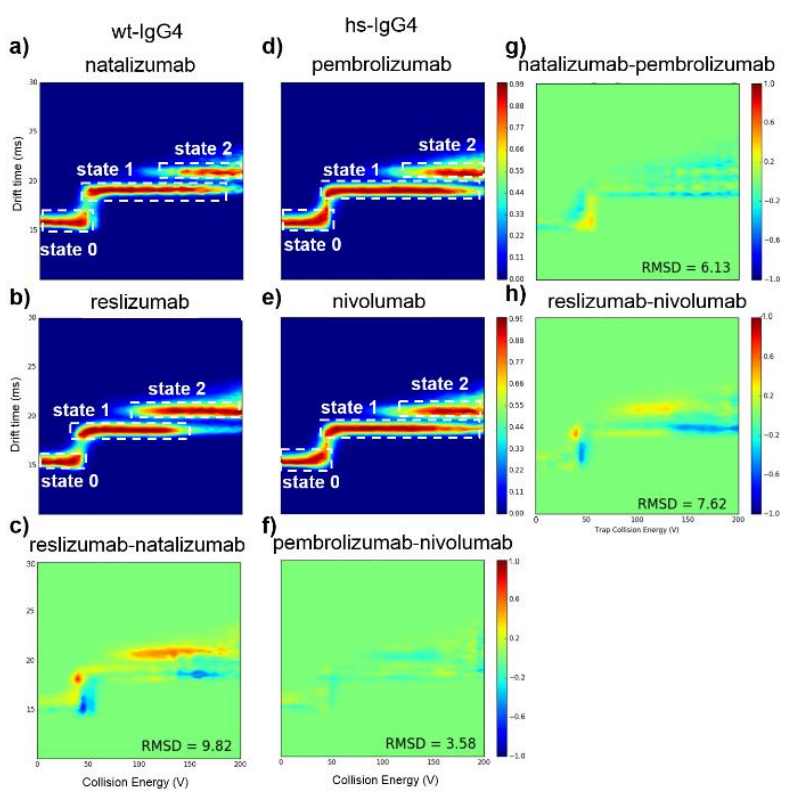

Figure 4. CIU fingerprints of the 22+ charge state of natalizumab (a), reslizumab (b), pembrolizumab (d), nivolumab (e), and differential CIU maps between reslizumab (red) and natalizumab (blue) (c), pembrolizumab (red) versus nivolumab (blue) (f), natalizumab (blue) vs pembrolizumab (red) (g), and reslizumab (blue) vs nivolumab (red) (h) from o to $200 \mathrm{~V}$ trap voltage.

Finally, we compared CIU patterns of wt- and hs-IgG4s, both containing four interchain disulfide bonds, but different hinge amino acid sequences and additional "flexibility/dynamics" in the hinge region of hs-IgG4 (Figure 1). An overall comparison of the CIU data acquired for these two $\mathrm{IgG}_{4}$ categories reveals many similarities. But, after detailed analysis, differences concerning the voltage associated with the unfolding transition can be distinguished. As previously observed in the wt- $\operatorname{IgG} 4$ differentiation, no significant differences are observed in the low voltage region (between $\mathrm{o}$ and $7 \mathrm{o} \mathrm{V}$ ), as the initial compact state $o$ of all $\mathrm{mAbs}$ has an IM drift time of between 15.5 and $16.1 \mathrm{~ms}$, with the first unfolding process also occurring between 35 and $40 \mathrm{~V}$ for the 22+ charge state of all mAbs (Figures 4 and 5). Most significant differences between wt- and hs-IgG4s can be observed in the high voltage region for the second conformational transition from unfolded state 1 to unfolded state 2 (Figure 5). At 140 $\mathrm{V}$, the relative intensity of the state 2 of the two wt-IgG4S is $49 \%$ and $58 \%$ for natalizumab and reslizumab, respectively, which is higher compared to the relative intensities of the state 2 for hs-IgG4S $(42 \%$ for both pembrolizumab and nivolumab). These observations emphasize that the second unfolding transition is slower for hs-IgG4s than for wt- mAbs, in agreement with a better resistance to gas-phase unfolding for hs-IgG4 formats compared to wt ones. 

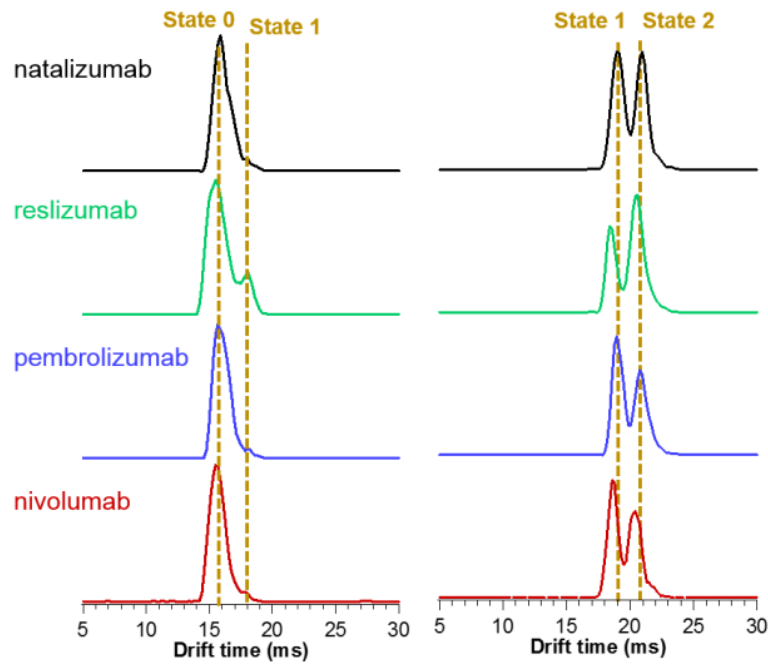

Figure 5. Extracted ATDs of 22+ charge state of wt- and hs-IgG4s at different collision energies (40 and $140 \mathrm{~V}$ ).

\section{IM-MS and CIU experiments to compare bsAb to former parent $\mathrm{wt}-\mathrm{IgG}_{4} \mathbf{m A b s}$}

We next focused on the IM-MS investigation of the bsAb formed between wt-IgG4s. Under our experimental conditions, IM-MS assays resulted in a ${ }^{{ }^{T W}} \mathrm{CCS}_{\mathrm{N}_{2}}$ measurement of $69.9 \mathrm{~nm}^{2}$ for the $22+$ charge state of natalizumab/reslizumab bsAb. Of note, the ${ }^{{ }^{T W}} \mathrm{CCS}_{\mathrm{N}_{2}}$ of bsAb corresponds to the average ${ }^{{ }^{T W}} \mathrm{CCS}_{\mathrm{N}_{2}}$ of natalizumab and reslizumab (70.1 and $69.6 \mathrm{~nm}^{2}$, respectively), which leads to the conclusion that no significant global structure modifications are induced through FAE reaction. Theoretical calculations, based on the empirical ${ }^{\mathrm{TW}} \mathrm{CCS}_{\mathrm{N}_{2}}$ equation for globular proteins, estimated an increase of 0.3 $\mathrm{nm}^{2}$ for reslizumab ${ }^{\mathrm{TW}} \mathrm{CCS}_{\mathrm{N}_{2}}$ after the addition of the HL pair of natalizumab, regardless of the charge state of the different species, which perfectly matches our experimental results $\left(\Delta^{\mathrm{TW}} \mathrm{CCS}_{\mathrm{N}_{2}}\right.$ (natalizumab/reslizumab) $=0.3 \pm 0.1 \mathrm{~nm}^{2}$ )

We next generated the CIU fingerprint of the newly formed $\mathrm{bsAb}$ in order to obtain more structural insights into the interaction between the two H-L pairs after FAE reaction (Figure 6a). As previously observed for all the $\mathrm{IgG}_{4}$ formats, the CIU fingerprint exhibits two unfolding transitions and three different conformational states. The centroid IM drift time associated with the initial compact state of bsAb is $15.9 \mathrm{~ms}$; after ion activation, the corresponding IM drift time of the most unfolded structure is $20.5 \mathrm{~ms}$, leading to a relative ${ }^{\mathrm{TW}} \mathrm{CCS}_{\mathrm{N}_{2}}$ increase of $6.3 \%$. In spite of the overall similarity of the bsAb and the previous $\mathrm{IgG}_{4}$ fingerprints, the collision voltage of the unfolding transitions is not completely the same.

The IM drift time of the most intense ATD distribution of natalizumab, reslizumab and the bsAb were monitored as a function of the collision voltage in order to compare the gas-phase unfolding of the three IgG4s involved in FAE reaction (Figure $6 \mathrm{~b}$ and Supporting Information Figure
S6). All along the trap voltage range (o-20o V), the IM drift time of the bsAb, and therefore its ${ }^{\mathrm{TW}} \mathrm{CCS}_{\mathrm{N}_{2}}$, is significantly different and systematically in between the values of its two parental wt-IgG4s. Furthermore, the two bsAb unfolding transitions occur at $45 \mathrm{~V}$ (reslizumab $40 \mathrm{~V}$ and natalizumab $50 \mathrm{~V}$ ) and $140 \mathrm{~V}$ (reslizumab $120 \mathrm{~V}$ and natalizumab $160 \mathrm{~V}$ ), which means that both occur between the natalizumab and reslizumab unfolding transitions, highlighting that the global conformation and the gasphase unfolding of the newly formed bsAb are directly related to the structure and gas-phase stability of both parental mAbs.
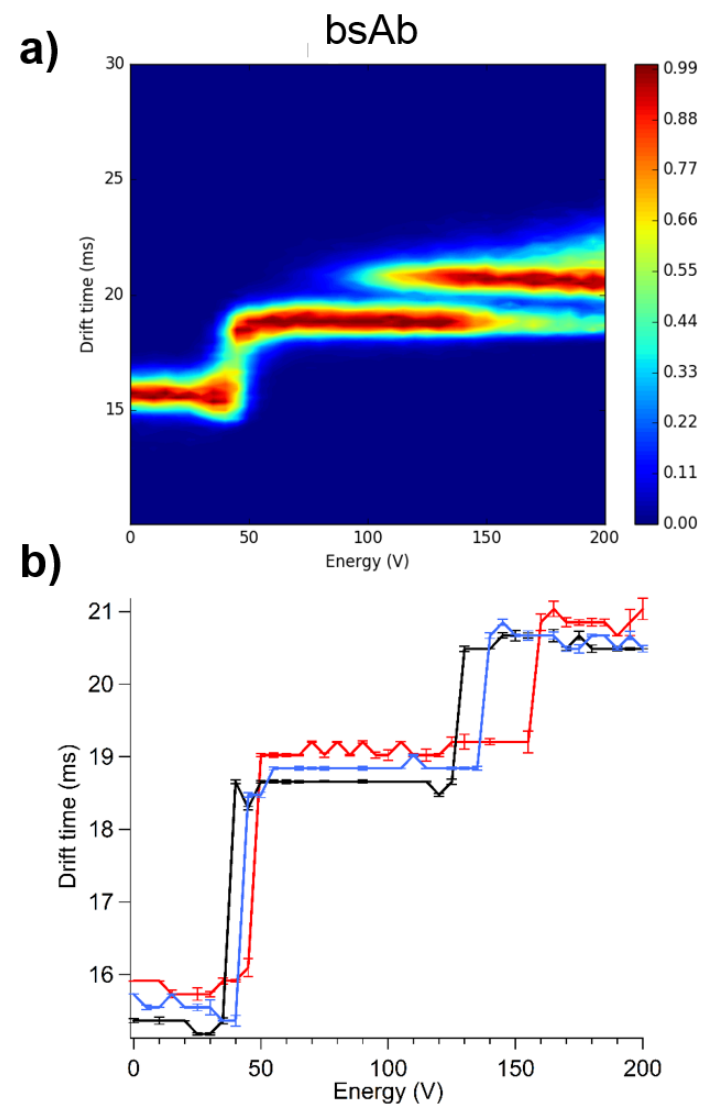

Figure 6. CIU of bsAb formed between natalizumab and reslizumab. (a) CIU fingerprint of the newly formed bsAb after FAE. (b) Evolution of the drift time of 22+ charge state of bsAb (blue), natalizumab (red), and reslizumab (black) as a function of the collision energy.

\section{Concluding remarks}

Here, we report on the complementarity of advanced CIU experiments with native MS and IM-MS in order to provide a global picture of the structure and gas-phase unfolding of IgG4-related $\mathrm{mAb}$ products. Native MS allows for the direct evaluation of the extent of FAE and the subsequent bsAb formation, which is a critical quality attribute requested by regulatory agencies in the approval of wt-IgG4 formats and as a negative control for FAE for hs-IgG4s. In addition, IM-MS approaches are foreseen to provide structural information on $\mathrm{mAb}$ structures. However, mAbs with identical or very closely related 
structures could co-migrate in classical IM-MS experiments, due to the fact that current IM drift cells do not provide the necessary resolving power to distinguish between subtle structural differences. This is the case in our study for $\mathrm{IgG}_{4} \mathrm{mAbs}$ with very similar primary sequences, as well as identical numbers and pairing of disulfide bonds, leading to very similar IM drift times and ${ }^{\mathrm{TW}} \mathrm{CCS}_{\mathrm{N}_{2}}$ values. Finally, CIU experiments performed either at the intact or middle (after IdeS enzymatic digestion) level enable the in-depth characterization and detailed differentiation of $\mathrm{IgG}_{4}$ formats. Activation of molecular ions prior to IM separation (CIU) has been proven to be a sensitive technique to assess the gas-phase stability of protein conformers ${ }^{30}$, and particularly to differentiate the four IgGk subclasses ${ }^{32}$. We focused here on one of these $\mathrm{mAb}$ isotype families $\left(\mathrm{IgG}_{4}\right)$ and took a closer look at the subtle differences that can exist within this family, by comparing CIU patterns of wt-, hs- and bsAb-IgG4s. Similar global CIU fingerprints were obtained for wt-, hsand bsAb-IgG4 formats, highlighting three main conformational states and two transitions. However, a detailed analysis of these gas-phase CIU fingerprints revealed small differences in terms of collision energies at which the unfolding transitions occur (higher energies for hs-IgG4), along with differences in the width of the energy range of the intermediate conformational states (unfolding state 1 of hs-IgG4 is more intense throughout the collision energy range). CIU experiments performed at the middle level after IdeS treatment allow concluding that $\mathrm{F}\left(\mathrm{ab}^{\prime}\right)_{2}$ domains drive the unfolding process of IgG4s. CIU was thus the only IM-MS based approach to unambiguously demonstrate the improved gas-phase stability of hs-IgG4, compared to other $\mathrm{IgG}_{4}$ formats, with hs-IgG4 mAbs (at both intact and middle level) being less prone to gas-phase unfolding, in agreement with less flexible hinge region scaffolds arising from the core-hinge S228P mutation. In the absence of three-dimensional structural data for most mAbs, CIU experiments appear to be an ingenious way to assess conformational rearrangements regarding small structural changes (protein isoforms ${ }^{30}$, chemical modifications ${ }^{31}$ etc.). Thus, CIU patterns can generate global isotypes fingerprints, as well as specific signatures of any individual $\mathrm{mAb}$.

The present study also focuses on the global conformational characterization of the bsAb formed upon FAE from two parent wt-IgG4s. Again, poor information can be deduced from the global structural IM-MS data, as similar ${ }^{\mathrm{TW}} \mathrm{CCS}_{\mathrm{N}_{2}}$ are recorded for both wt-IgG4 parents and for the newly formed bsAb. Conversely, CIU patterns appear to be more informative as the unfolding signature of the bsAb is intermediate to the ones of the former parent wt-IgG4s throughout the collision energy range, highlighting that the bsAb formed upon FAE keeps the memory of its origins. As for wt- and hs-IgG4s, bsAb CIU fingerprints present three different conformational states for which transitions energies represent the mean of parent wt-IgG4 ${ }^{\mathrm{TW}} \mathrm{CCS}_{\mathrm{N}_{2}}$.

Altogether our work presents an exhaustive landscape of the possibilities of native MS, IM-MS and CIU for the conformational characterization of the subfamily of $\operatorname{IgG}_{4}$ $\mathrm{mAb}$ formats. Native MS appears to be an alternative method to classical chromatographies ${ }^{42}$ used to monitor FAE, with the additional advantage of unambiguous mass identification of all detected species. Native MS, even when coupled to size exclusion chromatography for fast desalting $26-27,43$, provides, within 5-min runs, valuable information for $\mathrm{IgG}_{4}$ homogeneity characterization, including the possibility to monitor dynamics of FAE processes. While IM-MS appears to be poorly informative for the structural characterization of members of the $\mathrm{IgG}_{4}$ subfamily (wt-, hs- and bsAb-IgG4s), CIU experiments provide unique specific signatures of each individual $\mathrm{IgG}_{4}$, in turn providing resolution of these iso-cross-sectional species (which co-migrate in IM-MS) through their unfolding pathways. Common features of $\mathrm{IgG}_{4}$ formats include the observation of three conformational states and two transitions. Interestingly, $\mathrm{CIU}$ patterns of $\mathrm{F}\left(\mathrm{ab}^{\prime}\right)_{2}$ fragments obtained after IdeS digestion also proved to be $\mathrm{mAb}$-specific and can be seen as signatures/fingerprints of mAbs. In addition, CIU protocols are able to differentiate between stabilized and $w \mathrm{t}-\operatorname{IgG} 4 \mathrm{~s}$ and can enable the assessment of $\mathrm{mAb}$ stabilities. We believe that $\mathrm{CIU}$ experiments can be included in MS-based analytical workflows for the conformational/structural characterization of $\mathrm{mAb}$ formats in early development phases and in multiple attribute monitoring workflows.

\section{ASSOCIATED CONTENT}

\section{Supporting information}

Supporting Information Table S1, Table S2, Figure S1, Figure $\mathrm{S}_{2}$, Figure $\mathrm{S}_{3}$, Figure $\mathrm{S}_{4}$, Figure $\mathrm{S}_{5}$, and Figure $\mathrm{S}_{6}$ are available free of charge from the ACS Publications website.

\section{AUTHOR INFORMATION}

\section{Corresponding author}

*Phone: +33 (o)3 688526 79. Fax: +33 (o)3 68852781 .

E-mail: sarah.cianferani@unistra.fr

\section{Author contributions}

The manuscript was written with contributions from all authors. All authors have given approval to the final version of the manuscript.

\section{FUNDING SOURCES}

This work was supported by the CNRS, the Université de Strasbourg, the Agence Nationale de la Recherche and the French Proteomic Infrastructure (ANR-10-INBS-08-03).

\section{ACKNOWLEDGMENT}

The authors would like to thank GIS IBiSA and Région Alsace for financial support in purchasing a Synapt G2 HDMS instrument. O.A-H acknowledges the IdeX program of the University of Strasbourg for funding his postdoctoral fellowship.

\section{ABBREVIATIONS}

$\mathrm{mAb}$ monoclonal antibody

IgG immunoglobulin $\mathrm{G}$ 


$\begin{array}{ll}\text { FAE } & \text { fab-arm-exchange } \\ \text { CIU } & \text { collision induced unfolding } \\ \text { wt- } & \text { wild-type } \\ \text { hs- } & \text { hinge-stabilized } \\ \text { IMS } & \text { Ion mobility spectrometry } \\ \text { MS } & \text { Mass spectrometry } \\ \text { bsAb } & \text { bispecific antibody } \\ { }_{\text {TW }}{ }_{\text {CCS }}{ }_{\mathrm{N} 2} & \text { collision cross section measured in a } \\ & \text { TWIMS device with N2 as background } \\ & \text { gas } \\ \text { ATD } & \text { Arrival time distribution } \\ \text { F(ab') } 2 & \text { Full fragment antigen-binding } \\ \text { FC } & \text { Fragment cristallizable }\end{array}$

\section{REFERENCES}

1. Carter, P. J.; Lazar, G. A., Nature Reviews Drug Discovery 2018, 17 (3), 197-223.

2. Ecker, D. M.; Jones, S. D.; Levine, H. L., Mabs 2015, 7 (1), 9-14.

3. Denmark, V. K.; Mayer, L., Expert Review of Clinical Immunology 2013, 9 (1), 77-92.

4. Strohl, W. R., Protein \& Cell 2018, 9 (1), 86-120.

5. Kaplon, H.; Reichert, J. M., mAbs 2018, 1-21.

6. Beck, A.; Haeuw, J. F.; Wurch, T.; Goetsch, L.; Bailly, C.; Corvaia, N., Discovery Medicine 2010, 10 (53), 329-339.

7. Steiner, M.; Neri, D., Clinical Cancer Research 2011, 17 (20), 6406-6416.

8. Beck, A.; Reichert, J. M., Mabs 2011, 3 (3), 221-222.

9. Beck, A.; Wagner-Rousset, E.; Ayoub, D.; Van Dorsselaer, A.; Sanglier-Cianferani, S., Anal. Chem. 2013, 85 (2), 715-736.

10. Beck, A.; Goetsch, L.; Dumontet, C.; Corvaia, N., Nature Reviews Drug Discovery 2017, 16 (5), 315-337.

11. Zhang, A. M.; Fang, J.; Chou, R. Y. T.; Bondarenko, P. V.; Zhang, Z. Q., Biochemistry 2015, 54 (10), 1956-1962.

12. Beck, A.; Wurch, T.; Bailly, C.; Corvaia, N., Nature Reviews Immunology 2010, 10 (5), 345-352.

13. Vafa, O.; Gilliland, G. L.; Brezski, R. J.; Strake, B.; Wilkinson, T.; Lacy, E. R.; Scallon, B.; Teplyakov, A.; Malia, T. J.; Strohl, W. R., Methods 2014, 65 (1), 114-126.

14. Schuurman, J.; Parren, P., Current Opinion in Immunology 2016, 40, VII-XIII.

15. Goel, N.; Stephens, S., Mabs 2010, 2 (2), 137-147.

16. Cavaco, M.; Castanho, M. A. R. B.; Neves, V., Peptide Science 2018, 110 (1), e23095-n/a.

17. Sampei, Z.; Igawa, T.; Soeda, T.; Funaki, M.; Yoshihashi, K.; Kitazawa, T.; Muto, A.; Kojima, T.; Nakamura, S.; Hattori, K., Mabs 2015, 7 (1), 120-128.

18. Angal, S.; King, D. J.; Bodmer, M. W.; Turner, A.; Lawson, A. D. G.; Roberts, G.; Pedley, B.; Adair, J. R., Molecular Immunology 1993, 30 (1), 105-108.

19. Labrijn, A. F.; Buijsse, A. O.; van den Bremer, E. T. J.; Verwilligen, A. Y. W.; Bleeker, W. K.; Thorpe, S. J.; Killestein, J.; Polman, C. H.; Aalberse, R. C.; Schuurman, J.; van de Winkel, J. G. J.; Parren, P., Nat. Biotechnol. 2009, 27 (8), 767-U122.

2o. Rose, R. J.; Labrijn, A. F.; van den Bremer, E. T. J.; Loverix, S.; Lasters, I.; van Berkel, P. H. C.; van de Winkel, J. G. J.; Schuurman, J.; Parren, P.; Heck, A. J. R., Structure 2011, 19 (9), 1274-1282.

21. Kolfschoten, M. V.; Schuurman, J.; Losen, M.; Bleeker, W. K.; Martinez-Martinez, P.; Vermeulen, E.; den Bleker, T. H.;
Wiegman, L.; Vink, T.; Aarden, L. A.; De Baets, M. H.; van De Winkel, J. G. J.; Aalberse, R. C.; Parren, P., Science 2007, 317 (5844), 1554-1557.

22. Yang, X.; Wang, F.; Zhang, Y.; Wang, L.; Antonenko, S.; Zhang, S.; Zhang, Y. W.; Tabrizifard, M.; Ermakov, G.; Wiswell, D.; Beaumont, M.; Liu, L.; Richardson, D.; Shameem, M.; Ambrogelly, A., J. Pharm. Sci. 104 (12), 4002-4014.

23. Scapin, G.; Yang, X.; Prosise, W. W.; McCoy, M.; Reichert, P.; Johnston, J. M.; Kashi, R. S.; Strickland, C., Nature Structural \&Amp; Molecular Biology 2015, 22, 953.

24. Tan, S.; Zhang, H.; Chai, Y.; Song, H.; Tong, Z.; Wang, Q.; Qi, J.; Wong, G.; Zhu, X.; Liu, W. J.; Gao, S.; Wang, Z.; Shi, Y.; Yang, F.; Gao, G. F.; Yan, J., Nature Communications 2017, 8, 14369.

25. Couzin-Frankel, J., Science 2013, 342 (6165), 1432-1433.

26. Ehkirch, A.; Hernandez-Alba, O.; Colas, O.; Beck, A.; Guillarme, D.; Cianférani, S., J. Chromatogr. B 2018, 1086, 176-183.

27. Ehkirch, A.; D'Atri, V.; Rouviere, F.; Hernandez-Alba, O.; Goyon, A.; Colas, O.; Sarrut, M.; Beck, A.; Guillarme, D.; Heinisch, S.; Cianferani, S., Anal. Chem. 2018, 90 (3), 1578-1586.

28. Beck, A.; Terral, G.; Debaene, F.; Wagner-Rousset, E.; Marcoux, J.; Janin-Bussat, M.-C.; Colas, O.; Dorsselaer, A. V.; Cianférani, S., Expert Review of Proteomics 2016, 13 (2), 157-183.

29. Beck, A.; Sanglier-Cianférani, S.; Van Dorsselaer, A., Anal. Chem. 2012, 84 (11), 4637-4646.

3o. Eschweiler, J. D.; Martini, R. M.; Ruotolo, B. T., J. Am. Chem. Soc. 2017, 139 (1), 534-540.

31. Dixit, S. M.; Polasky, D. A.; Ruotolo, B. T., Curr. Opin. Chem. Biol. 2018, 42, 93-100.

32. Tian, Y. W.; Han, L. J.; Buckner, A. C.; Ruotolo, B. T., Anal. Chem. 2015, 87 (22), 11509-11515.

33. Debaene, F.; Wagner-Rousset, E.; Colas, O.; Ayoub, D.; Corvaia, N.; Van Dorsselaer, A.; Beck, A.; Cianferani, S., Anal. Chem. 2013, 85 (20), 9785-9792.

34. Bush, M. F.; Hall, Z.; Giles, K.; Hoyes, J.; Robinson, C. V.; Ruotolo, B. T., Anal. Chem. 2010, 82 (22), 9557-9565.

35. Eschweiler, J. D.; Rabuck-Gibbons, J. N.; Tian, Y. W.; Ruotolo, B. T., Anal. Chem. 2015, 87 (22), 11516-11522.

36. Stubenrauch, K.; Wessels, U.; Regula, J. T.; Kettenberger, H.; Schleypen, J.; Kohnert, U., Drug Metabolism and Disposition 2010, 38 (1), 84-91.

37. Lewis, K. B.; Meengs, B.; Bondensgaard, K.; Chin, L.; Hughes, S. D.; Kjaer, B.; Lund, S.; Wang, L. P., Molecular Immunology 2009, 46 (16), 3488-3494.

38. Ruotolo, B. T.; Benesch, J. L. P.; Sandercock, A. M.; Hyung, S.-J.; Robinson, C. V., Nature Protocols 2008, 3, 1139.

39. Allen, S. J.; Schwartz, A. M.; Bush, M. F., Anal. Chem. 2013, 85 (24), 12055-12061.

40. Botzanowski, T.; Erb, S.; Hernandez-Alba, O.; Ehkirch, A.; Colas, O.; Wagner-Rousset, E.; Rabuka, D.; Beck, A.; Drake, P. M.; Cianférani, S., mAbs 2017, 9 (5), 801-811.

41. Chevreux, G.; Tilly, N.; Bihoreau, N., Anal. Biochem. 2011, 415 (2), 212-214.

42. $\quad$ Yang, X.; Zhang, Y.; Wang, F.; Wang, L.; Richardson, D.; Shameem, M.; Ambrogelly, A., Anal. Biochem. 2015, 484, 173-179.

43. Van der Rest, G.; Halgand, F., J. Am. Soc. Mass. Spectrom. 2017, 28 (11), 2519-2522. 


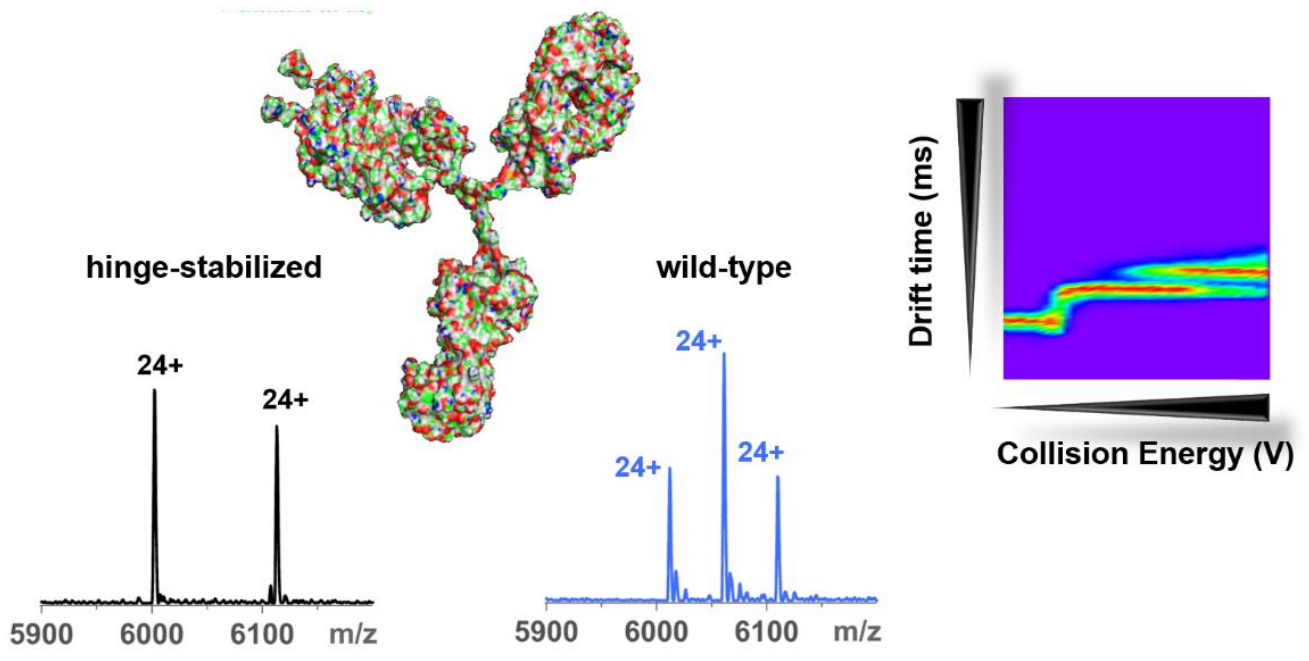

